\title{
Electron energy loss and induced photon emission in photonic crystals
}

\author{
F. J. García de Abajo ${ }^{1,2 *}$ and L. A. Blanco ${ }^{2}$ \\ ${ }^{1}$ Centro Mixto CSIC-UPV/EHU, Apartado Postal 1072, 20080 San Sebastián, Spain \\ ${ }^{2}$ Donostia International Physics Center (DIPC), Apartado Postal 1072, 20080 San Sebastián, Spain
}

(Received 14 October 2002; published 14 March 2003)

\begin{abstract}
The interaction of a fast electron with a photonic crystal is investigated by solving the Maxwell equations exactly for the external field provided by the electron in the presence of the crystal. The energy loss is obtained from the retarding force exerted on the electron by the induced electric field, and the photon emission probability is calculated from the far-field Poynting vector. The features of the energy-loss spectra are shown to be related to the photonic band structure of the crystal. Two different regimes are discussed: for small lattice constants $a$ relative to the wavelength of the associated electron excitations $\lambda$, an effective medium theory can be used to describe the material; however, for $a \sim \lambda$ the photonic band structure plays an important role. Special attention is paid to the frequency gap regions in the latter case.
\end{abstract}

DOI: 10.1103/PhysRevB.67.125108

PACS number(s): 41.75.Ht, 79.20.Kz, 41.20.Jb, 03.50.De

\section{INTRODUCTION}

Near-field spectroscopy can be performed using spatially resolved electron energy-loss spectroscopy in scanning transmission electron microscopes, where the electron beam provides an external evanescent field to probe the sample within a spatial range on the nanometer scale. In particular, the relatively intense, low-energy part $(<50 \mathrm{eV})$ of the loss spectrum can be used with minimum sample damage to provide some insight on plasmons and other collective excitations. ${ }^{1-4}$ For the electron velocities typically employed in microscopes (above half the speed of light) and for samples that are homogeneous across distances of a few nanometers, frequency-dependent dielectric functions are sufficient to describe the materials that are involved and the loss spectra reflect the geometry of the sample interfaces. ${ }^{5-10}$

The geometry becomes particularly important in photonic crystals, where the periodic spatial modulation of the dielectric function affects the propagation of radiation to the point of forbidding it within band-gap energy regions. As a consequence, photonic crystals are known to inhibit the spontaneous emission of light within the band gap. ${ }^{11,12}$ They can also be used to make omnidirectional dielectric mirrors that reflect light from all directions without absorption ${ }^{13}$ and wave guides able to deflect light around sharp corners on the scale of the wavelength. ${ }^{14}$ These are applications of photonic crystals that involve free external radiation, but equally remarkable effects are expected to accompany evanescent fields like those of external electrons.

In this work, we examine the energy-loss spectra of electrons moving near or inside photonic crystals over a wide range of lattice parameters $a$. When $a$ is much smaller than the wavelength of the radiation associated to a given energy loss, $\lambda$, it is shown that the crystal can be described to some extend by an effective dielectric function. ${ }^{15-18}$ For larger lattice constants, the photonic band structure becomes more complicated and this is reflected in the loss spectra. The relation between the band structure, the reflectance of photonic crystal slabs, and the electron energy-loss spectra is discussed in detail in what follows.

In a previous development, Pendry and Martín-Moreno ${ }^{19}$ calculated the energy-loss probability for electrons moving near crystals made of either metallic spheres or metallic cylinders in the $a \ll \lambda$ limit using the transfer-matrix approach ${ }^{20}$ to solve Maxwell's equations. Their results exhibit a complicated energy-loss structure even for relatively dilute crystals, which has not been reproduced in the present work. Therefore, in order to test the convergence of our method, we have calculated the energy loss near the surface of a crystal of dilute spheres and have found very good agreement between the results derived from the theory described in this work and those obtained from the analytical expression for isolated spheres. ${ }^{9,10}$

In addition to producing energy loss, the interaction between the electron and the crystal gives rise to the emission of the so-called Smith-Purcell radiation. ${ }^{21}$ This effect has already been discussed for one-dimensional ${ }^{10}$ and threedimensional ${ }^{22,23}$ crystals. Radiation emission is one of the contributions to the total energy loss, and in frequency regions where the crystal is transparent the probability of these two must coincide. Examples of this are offered below.

Here, the energy-loss and photon emission probability are calculated from the induced electric field, which is derived using the reflection coefficients of the crystal,,${ }^{19}$ as explained in Sec. II. Results for the case of small lattice constants ( $a$ $\ll \lambda)$ are given in Sec. III and for larger ones $(a \sim \lambda)$ in Secs. IV and V. The main conclusions are summarized in Sec. VI. Gaussian atomic units (a.u., that is, $\hbar=m=e=1$ ) will be used from now on, unless otherwise specified.

\section{THEORY}

\section{A. The reflection coefficients of the crystal}

We shall consider crystals that are composed of a number of layers perpendicular to the $z$ direction and extending from $z=0$ towards $z<0$. The host region outside the crystal will be assumed to be described by a frequency-dependent dielectric function $\epsilon_{h}(\omega)$ and a magnetic permeability $\mu_{h}(\omega)$. Each crystal layer consists of the repetition of a given object with certain two-dimensional (2D) translational symmetry that is shared by all layers. The crystal will be characterized here by its reflection coefficients, that is, the amplitudes of the reflected plane-wave components for a given incident wave. 
In general, an external plane wave of frequency $\omega$ that propagates near the crystal can be represented as

$$
\mathbf{E}_{\mathbf{Q}}^{ \pm} \exp \left(\mathrm{i} \mathbf{K}_{\mathbf{Q}}^{ \pm} \cdot \mathbf{r}\right)
$$

where $\mathbf{Q}=\left(Q_{x}, Q_{y}\right)$,

$$
\begin{gathered}
\mathbf{K}_{\mathbf{Q}}^{ \pm}=\left(\mathbf{Q}, \pm \mathrm{i} \Gamma_{Q}\right), \\
\Gamma_{Q}^{2}=Q^{2}-k_{h}^{2},
\end{gathered}
$$

and $k_{h}=(\omega / c)\left(\epsilon_{h} \mu_{h}\right)^{1 / 2}$ is the momentum of the plane wave. The real part of $\Gamma_{Q}$ is chosen to be positive, and the $+(-)$ sign in these expressions stands for a wave moving towards $z>0(z<0) .^{24}$

The momentum $\mathbf{Q}$ parallel to the surface has been singled out to make explicit use of crystal symmetry: invoking momentum conservation, a wave incident from the $z>0$ region with momentum $\mathbf{K}_{\mathbf{Q}}^{-}$will only produce a discrete set of reflected waves of momentum $\mathbf{K}_{\mathbf{Q}+\mathbf{G}}^{+}$, where $\mathbf{G}$ runs over reciprocal surface lattice vectors.

The transversal character of these waves (i.e., the fact that $\mathbf{K}_{\mathbf{Q}}^{ \pm} \cdot \mathbf{E}_{\mathbf{Q}}^{ \pm}=0$ ) can be exploited to express the electric field in terms of $s$ and $p$ components according to

$$
\mathbf{E}_{\mathbf{Q}}^{ \pm}=E_{\mathbf{Q}, s}^{ \pm} \hat{\mathbf{e}}_{\mathbf{Q}, s}^{ \pm}+E_{\mathbf{Q}, p}^{ \pm} \hat{\mathbf{e}}_{\mathbf{Q}, p}^{ \pm},
$$

where the vectors

$$
\hat{\mathbf{e}}_{\mathbf{Q}, s}^{ \pm}=\frac{1}{Q}\left(-Q_{y}, Q_{x}, 0\right)
$$

and

$$
\hat{\mathbf{e}}_{\mathbf{Q}, p}^{ \pm}=\frac{\mathrm{i}}{k_{h} Q}\left( \pm \Gamma_{Q} Q_{x}, \pm \Gamma_{Q} Q_{y}, \mathrm{i} Q^{2}\right)
$$

satisfy the identities $\hat{\mathbf{e}}_{\mathbf{Q}, s}^{ \pm} \cdot \hat{\mathbf{e}}_{\mathbf{Q}, s}^{ \pm}=\hat{\mathbf{e}}_{\mathbf{Q}, p}^{ \pm} \cdot \hat{\mathbf{e}}_{\mathbf{Q}, p}^{ \pm}=1$ and $\hat{\mathbf{e}}_{\mathbf{Q}, s}^{ \pm} \cdot \hat{\mathbf{e}}_{\mathbf{Q}, p}^{ \pm}$ $=\hat{\mathbf{e}}_{\mathbf{Q}, s}^{ \pm} \cdot \mathbf{K}_{\mathbf{Q}}^{ \pm}=\hat{\mathbf{e}}_{\mathbf{Q}, p}^{ \pm} \cdot \mathbf{K}_{\mathbf{Q}}^{ \pm}=0$. Also, $\hat{\mathbf{e}}_{\mathbf{Q}, s}^{ \pm}$is perpendicular to the plane defined by $\mathbf{Q}$ and the surface normal.

The amplitudes of the reflected waves depend linearly on the amplitudes of $s$ and $p$ components of the incident wave, and the coefficients of the linear relation between them are the reflection coefficients $R_{\mathbf{Q G}}^{\sigma \sigma^{\prime}}$, implicitly defined by

$$
\left[E_{\mathbf{Q}+\mathbf{G}, \sigma}^{+}\right]^{r}=\sum_{\sigma^{\prime}} R_{\mathbf{Q G}}^{\sigma \sigma^{\prime}}\left[E_{\mathbf{Q}, \sigma^{\prime}}^{-}\right]^{i},
$$

where $\sigma$ and $\sigma^{\prime}$ run over polarization directions $s$ and $p$, and the superindices $r$ and $i$ stand for reflected and incident components, respectively.

We have used the layer Korringa-Kohn-Rostoker (KKR) method to calculate the reflection coefficients $R_{\mathbf{Q G}}^{\sigma \sigma^{\prime}}$ both for $a \ll \lambda$ and for $a \sim \lambda$. In the layer KKR method, the transmission and reflection coefficients are calculated exactly for each single layer using multiple scattering in a basis set of multipoles centered around each object of the layer. Scattering among layers is then expressed in terms of those coefficients. The maximum multipole order $l_{\max }$ and the number of reflected and transmitted beams (i.e., the number of $\mathbf{G}$ vectors) are the only convergence parameters, which have been tested in all calculated results shown below. Stefanou et al. ${ }^{25}$ developed this method for spheres and we have extended it to be able to deal with nonspherical objects and arbitrary values of $l_{\max }$. Further details of the method will be given elsewhere. $^{26}$

\section{B. The field of the electron}

We shall consider an electron moving with constant velocity $v$ along a trajectory parallel to the crystal surface and described by $\mathbf{r}_{t}=\left(v t, y_{0}, z_{0}\right)$, with $z_{0}>0$, so that the electron moves in the host medium described by $\epsilon_{h}$ and $\mu_{h}$. Neglecting the crystal for the moment, the electron electric field can be expressed in frequency space $\omega$ in terms of vector and scalar potentials as

$$
\mathbf{E}_{0}=\frac{i \omega}{c} \mathbf{A}_{0}-\nabla \phi_{0}
$$

In the Lorentz gauge, Maxwell's equations can be recast as

$$
\left(\nabla^{2}+k_{h}^{2}\right) \phi_{0}=-\frac{4 \pi}{\epsilon_{h}} \rho
$$

and

$$
\left(\nabla^{2}+k_{h}^{2}\right) \mathbf{A}_{0}=-\frac{4 \pi \mu_{h}}{c} \mathbf{j}
$$

where $\rho(\mathbf{r}, \omega)$ is the electron charge density, and $\mathbf{j}(\mathbf{r}, \omega)$ $=(v \rho, 0,0)$ is its charge current.

Using the relation

$$
\left(\nabla^{2}+k_{h}^{2}\right) \int \frac{d \mathbf{p}}{2 \pi^{2}} \frac{e^{\mathrm{i} \mathbf{p} \cdot \mathbf{r}}}{p^{2}-k_{h}^{2}-\mathrm{i} 0^{+}}=-4 \pi \delta(\mathbf{r})
$$

and noticing that $\rho(\mathbf{r}, t)=-\delta\left(\mathbf{r}-\left(v t, y_{0}, z_{0}\right)\right)$, the electric field is found to be

$$
\mathbf{E}_{0}(\mathbf{r}, \omega)=\left[\frac{\nabla}{\epsilon_{h}}-\frac{\mathrm{i} \omega v \mu_{h}}{c^{2}} \hat{\mathbf{x}}\right] \int \frac{d \mathbf{p}}{2 \pi^{2}} \int d t e^{\mathrm{i} \omega t} \frac{e^{\mathrm{i} \mathbf{p} \cdot\left[\mathbf{r}-\left(v t, y_{0}, z_{0}\right)\right]}}{p^{2}-k_{h}^{2}-\mathrm{i} 0^{+}}
$$

where the time integral represents the inverse Fourier transform that permits obtaining $\rho(\mathbf{r}, \omega)$ in terms of $\rho(\mathbf{r}, t)$. The above integral can be reduced to

$$
\mathbf{E}_{0}(\mathbf{r}, \omega)=\int d Q_{y} e^{i \mathbf{K}_{\mathbf{Q}}^{ \pm} \cdot\left[\mathbf{r}-\left(0, y_{0}, z_{0}\right)\right]} \mathbf{E}_{\mathbf{Q}}^{ \pm}
$$

where $\mathbf{Q}=\left(\omega / v, Q_{y}\right)$ and

$$
\mathbf{E}_{\mathbf{Q}}^{ \pm}=\frac{\mathrm{i}}{\Gamma_{Q}}\left(\frac{\mathbf{K}_{\mathbf{Q}}^{ \pm}}{v \epsilon_{h}}-\frac{\omega \mu_{h}}{c^{2}} \hat{\mathbf{x}}\right) .
$$

The $+(-)$ sign must be used in these expressions when $z$ $>z_{0}\left(z<z_{0}\right)$, so that the integrand of Eq. (2) represents a plane wave that propagates towards positive (negative) $z$ 's. When the electron is moving in vacuum, $\Gamma_{Q}$ is real and the waves in the integrand of Eq. (2) are evanescent. ${ }^{24}$ However, when $k_{h}$ is real and larger than $\omega / v$, some of those waves 
describe Cherenkov radiation that propagates without attenuation; this is the case of electrons that travel faster than light in the medium.

\section{The field induced by interaction of the electron and the crystal}

The decomposition of these plane waves into $s$ and $p$ components is readily found to be

$$
\left[E_{\mathbf{Q}, s}^{ \pm}\right]^{i}=\frac{\mathrm{i} Q_{y} \omega \mu_{h}}{Q \Gamma_{Q} c^{2}} e^{-i \mathbf{K}_{\mathbf{Q}}^{ \pm} \cdot\left(0, y_{0}, z_{0}\right)}
$$

and

$$
\left[E_{\mathbf{Q}, p}^{ \pm}\right]^{i}= \pm \frac{k_{h}}{v Q \epsilon_{h}} e^{-i \mathbf{K}_{\mathbf{Q}}^{ \pm} \cdot\left(0, y_{0}, z_{0}\right)} .
$$

Each of the incident plane waves [i.e., each value of $Q_{y}$ in Eq. (2)] gives rise to a set of reflected waves whose amplitudes are obtained from Eq. (1). Therefore, the electric field in the region near the ion can be constructed as the sum of $\mathbf{E}_{0}$ and the reflected field,

$$
\mathbf{E}(\mathbf{r}, \omega)=\mathbf{E}_{0}(\mathbf{r}, \omega)+\mathbf{E}_{r}(\mathbf{r}, \omega),
$$

where

$$
\mathbf{E}_{r}(\mathbf{r}, \omega)=\sum_{\mathbf{G}, \sigma \sigma^{\prime}} \int d Q_{y} e^{i \mathbf{K}_{\mathbf{Q}+\mathbf{G}}^{+} \cdot \mathbf{r}} R_{\mathbf{Q G}}^{\sigma \sigma^{\prime}}\left[E_{\mathbf{Q}, \sigma^{\prime}}^{-}\right]^{i} \hat{\mathbf{e}}_{\mathbf{Q}+\mathbf{G}, \sigma}^{+},
$$

and the integral over $Q_{y}$ has been copied directly from Eq. (2) in virtue of the linearity of Maxwell's equations.

\section{Electron energy loss in front of the crystal}

The electron energy loss can be calculated from the retarded force exerted by the induced part of the electric field $\mathbf{E}^{\text {ind }}$ acting back on the electron. Integrating this force along the trajectory and dividing by the total path length $L$, one finds

$$
\frac{\Delta E}{\Delta x}=\frac{v}{L} \int d t E_{x}^{\mathrm{ind}}\left(\mathbf{r}_{t}, t\right)=\int_{0}^{\infty} \omega d \omega P(\omega),
$$

where

$$
P(\omega)=\frac{v}{\pi \omega L} \int d t \operatorname{Re}\left\{e^{-\mathrm{i} \omega t} E_{x}^{\mathrm{ind}}\left(\mathbf{r}_{t}, \omega\right)\right\}
$$

is the loss probability per unit of path length.

For an electron moving parallel to a crystal surface, Eq. (3) permits separating the loss probability as

$$
P=P_{0}+P_{r},
$$

where $^{9}$

$$
P_{0}(\omega)=\frac{1}{\pi v^{2}} \operatorname{Im}\left\{\left(\frac{v^{2}}{c^{2}} \mu_{h}-\frac{1}{\epsilon_{h}}\right) \ln \left[\frac{q_{c}^{2}-k_{h}^{2}}{(\omega / v)^{2}-k_{h}^{2}}\right]\right\}
$$

corresponds to the contribution of $\mathbf{E}_{0}$ in the absence of the crystal. Here, $q_{c}$ is a momentum cutoff related to energy conservation. This contribution vanishes in vacuum. However, it gives rise to Cherenkov losses when $\epsilon_{h}$ and $\mu_{h}$ are real, in which case ${ }^{27,9} P_{0}=\left|\mu_{h}\right|\left[1 / c^{2}-1 /\left(v^{2} \epsilon_{h} \mu_{h}\right)\right]$, subject to the Cherenkov condition $v^{2} \epsilon_{h} \mu_{h}>c^{2}$.

The remaining contribution $P_{r}$ is due to the retarding force exerted by the reflected field $\mathbf{E}_{r}$. Inserting Eq. (4) into Eq. (5), the time $t$ and the lateral impact parameter $y_{0}$ appear only through the factor $\exp \left\{i\left(G_{x} v t+G_{y} y_{0}\right)\right\}$, so that time integration eliminates all vectors $\mathbf{G}$ with $G_{x} \neq 0$ from the sum in Eq. (4). Furthermore, averaging over $y_{0}$ leaves only the $G_{y}=0$ term and one obtains

$$
\begin{aligned}
P_{r}(\omega)= & \frac{1}{\pi v^{2}} \int \frac{d Q_{y}}{Q^{2}} \operatorname{Im}\left\{\left[R_{\mathbf{Q} 0}^{p p}+\epsilon_{h} \mu_{h}\left(\frac{Q_{y} v}{\Gamma_{Q} c}\right)^{2} R_{\mathbf{Q} 0}^{s s}\right.\right. \\
& \left.\left.+\frac{\mathrm{i} Q_{y} k_{h} v}{\omega \Gamma_{Q}}\left(R_{\mathbf{Q} 0}^{s p}-R_{\mathbf{Q} 0}^{p s}\right)\right] \frac{\Gamma_{Q} e^{-2 \Gamma_{Q^{z} 0}}}{\epsilon_{h}}\right\} .
\end{aligned}
$$

The last term inside the square bracket of this expression gives no contribution when the electron trajectory is contained in a plane of specular symmetry of the crystal surface.

As a test one can apply Eq. (6) to an electron moving in vacuum $\left(\epsilon_{h}=\mu_{h}=1\right)$ at a distance $z_{0}$ from the surface of a nonmagnetic medium described by $\epsilon$, in which case $R_{\mathbf{Q} 0}^{s p}$ $=R_{\mathbf{Q} 0}^{p s}=0$. Then, using the fact that $P_{0}=0$ in vacuum, so that $P=P_{r}$, Eq. (6) leads to

$$
\begin{aligned}
P(\omega)= & \frac{2}{\pi v^{2}} \int_{0}^{\infty} \frac{\Gamma_{Q} d Q_{y}}{Q^{2}} e^{-2 \Gamma_{Q} z_{0}} \operatorname{Im}\left\{\frac{\epsilon \Gamma_{Q}-\Gamma_{Q}^{\prime}}{\epsilon \Gamma_{Q}+\Gamma_{Q}^{\prime}}\right. \\
& \left.+\left(\frac{Q_{y} v}{\Gamma_{Q} c}\right)^{2} \frac{\Gamma_{Q}-\Gamma_{Q}^{\prime}}{\Gamma_{Q}+\Gamma_{Q}^{\prime}}\right\},
\end{aligned}
$$

where $\Gamma_{Q}^{\prime}=\sqrt{Q^{2}-\epsilon \omega^{2} / c^{2}}, \Gamma_{Q}=\sqrt{Q^{2}-\omega^{2} / c^{2}}$, and the reflection coefficients of Eq. (6) have been taken from Fresnel's equations. ${ }^{27}$ In the nonrelativistic limit, $\Gamma_{Q}=\Gamma_{Q}^{\prime}$ $=Q$, so that the contribution of the reflection of $s$ waves vanishes and the loss probability becomes proportional to $\operatorname{Im}\{-1 /(\epsilon+1)\}$.

\section{ENERGY LOSS FOR SMALL LATTICE CONSTANTS}

When the crystal lattice constant is much smaller than the wavelength corresponding to a given frequency component $\omega$, the details of the crystal lattice cannot be resolved by the electron, so that the medium behaves like a uniform material, characterized by an effective dielectric constant and magnetic permeability. For instance, a dilute system of spheres can be regarded as a set of interacting dipoles, which leads to the well-known Maxwell-Garnett formula. ${ }^{28}$ For more compact systems the details of the microscopic structure becomes relevant via important multipolar interactions.

These are the cases considered in Fig. 1 for a crystal composed of small aluminum spheres that are disposed in a simple cubic lattice of constant $a=5 \mathrm{~nm}$. The solid curves of Fig. 1(a) show the loss probability for an electron moving 


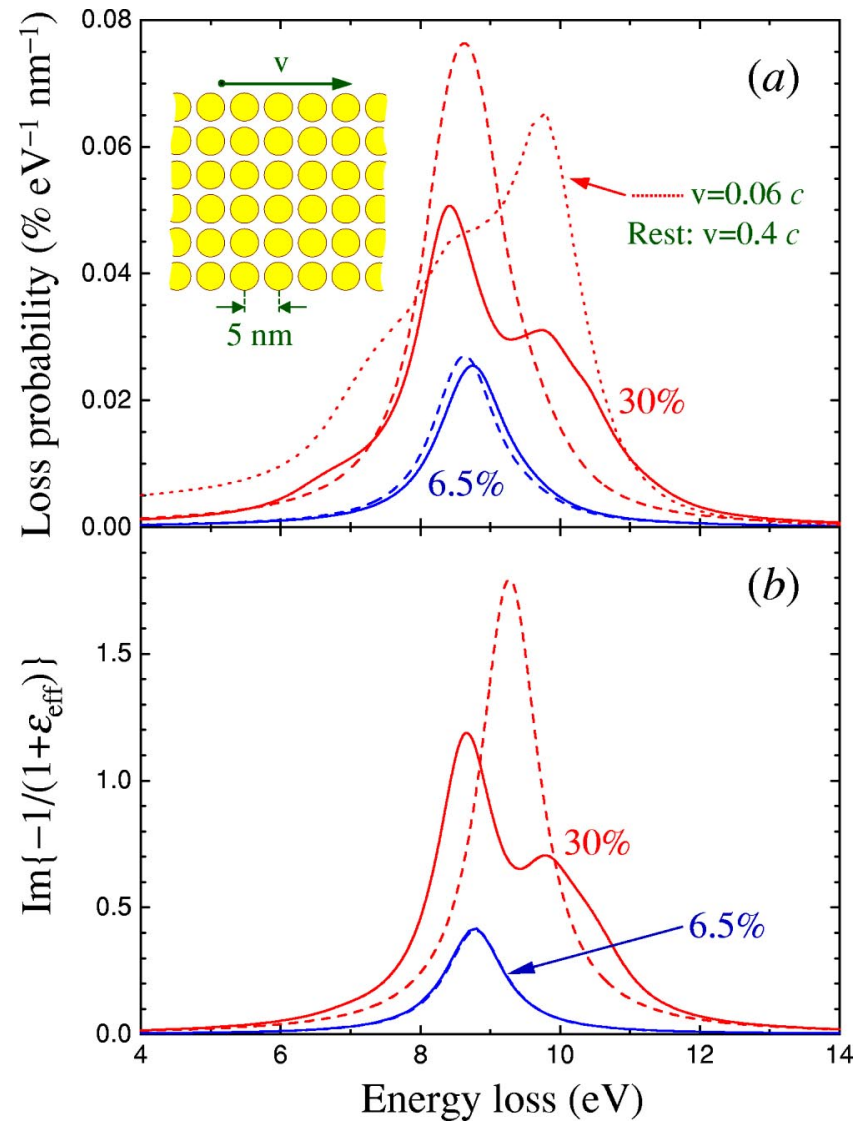

FIG. 1. (color online only) (a) Energy-loss spectra for an electron moving parallel to the [100] direction of the (100) surface of a simple cubic crystal made up of six layers of aluminum spheres in vacuum with lattice constant $5 \mathrm{~nm}$ and two different filling fractions (see labels). The electron is moving at a distance of $1 \mathrm{~nm}$ from the sphere surfaces with a velocity $v=0.4 c$ (solid and dashed curves) and $v=0.06 c$ (dotted curve). The dashed curves are obtained by neglecting the interaction among spheres, whereas the solid curves and the dotted curve correspond to the full solution of Maxwell's equations. (b) Surface loss function for the same crystal as in (a) using the effective dielectric function obtained from the MaxwellGarnett expression (broken curves) and from the multiple-scattering method explained in the text (solid curves).

with velocity $v=0.4 c$ parallel to the surface of a slab made up of six (100) layers of such crystal. The lost probability has been calculated by means of Eq. (6) for two different filling fractions $f$ of the aluminum $(6.5 \%$ and $30 \%$, respectively). The dielectric function of aluminum has been approximated by a Drude expression $\epsilon(\omega)=1-\omega_{p}^{2} / \omega(\omega$ $+\mathrm{i} \eta)$ with $\omega_{p}=15 \mathrm{eV}$ and $\eta=1 \mathrm{eV}$. The wavelengths considered in the figure lie in the range $\lambda=2 \pi c / \omega$ $=88.6-310 \mathrm{~nm}$, so that we are in the $\lambda \gg a$ limit. The maximum orbital quantum number used to achieve convergence on the figure is $l_{\max }=6$.

Crystalline effects come from the interaction among spheres, and a method to determine their relative role consists in comparing these results with a calculation in which that interaction is suppressed. This is what the dashed curves stand for in Fig. 1. It is very clear that the interaction among spheres is almost negligible for $f=6.5 \%$, whereas it be- comes very important at $f=30 \%$. In the latter case, the sphere surfaces are closer to each other and their mutual electromagnetic coupling becomes relevant. The noninteracting case shows a peak at around $\omega_{1}=8.7 \mathrm{eV}$ that corresponds to the dipole Mie resonance in the small-sphere limit, given by the expression $\epsilon\left(\omega_{1}\right)=-2$ (i.e., $\left.\omega_{1}=\omega_{p} / \sqrt{3}\right)$. The main effect of the sphere-sphere interaction (solid curve for $f=30 \%$ ) consists in splitting this peak, in a similar way as splitting of degenerate levels (the Mie resonances here) occurs in atomic bonding.

This is actually observed in the projected photonic band structure of this crystal, represented in Fig. 2 for $f=30 \%$. The structure is dominated by nearly flat bands corresponding to localized excitations near the Mie resonances of the small isolated spheres, $\omega_{l}=\omega_{p} \sqrt{l /(2 l+1)}$, which lie in the 8.7-10.6 eV range (see the labels on the right-hand side of the figure for the dipole and quadrupole Mie modes). The interaction between spheres gives rise to a complex structure that encompasses regions of relative transparency outside that energy range. The evanescent plane-wave components of the external electron field are subject to the condition $\omega$ $=\mathbf{Q} \cdot \mathbf{v}$, which defines the solid straight lines shown in the figure. The loss spectrum can then be understood as originating from both absorption and direct coupling to propagating modes of the crystal. This last effect is clearly seen as a bump in the loss probability near $6.7 \mathrm{eV}$, connected to the crossing of the noted straight lines with a low-energy region of allowed propagating modes.

The dashed curves of Fig. 1(a) (uncoupled spheres) have been obtained in two different ways. The first one consists in calculating the reflection coefficients that appear in Eq. (6) using the layer KKR method but neglecting the interaction among spheres within each layer and also the interaction among different layers. A second procedure consists in making use of the analytical expression for the energy-loss probability of an electron near an isolated sphere (see Refs. 6, 9, and 10 for non relativistic and fully relativistic formulas, respectively) and summing over all impact parameters of the electron trajectory with respect to the spheres of the crystal. The results coming out of these two very different procedures cannot be distinguished on the scale of the figure, and this is a strong indication of the degree of convergence of our numerical calculations with respect to the number of plane waves used in the layer KKR method.

These results do not support, however, previous calculations by Pendry and Martín-Moreno ${ }^{19}$ for this exact system, where the number of features and their energy position for the filling fractions under consideration differ from ours. A possible lack of convergence of the transfer-matrix method used by the authors for this three-dimensional system of metallic scatterers might be the reason of this discrepancy. In particular, some of their low-energy features around $6.5 \mathrm{eV}$ could originate in the modes of the wedge associated to their space discretization procedure (resonances near that energy have also been found for the $90^{\circ}$ aluminum wedge ${ }^{9}$ ).

The example offered in Fig. 1 illustrates what happens in the $a \ll \lambda$ limit, where it is reasonable to define an effective dielectric function $\epsilon_{\text {eff }}$ for the infinite crystal and to compare the results of the detailed, exact calculation with those ob- 


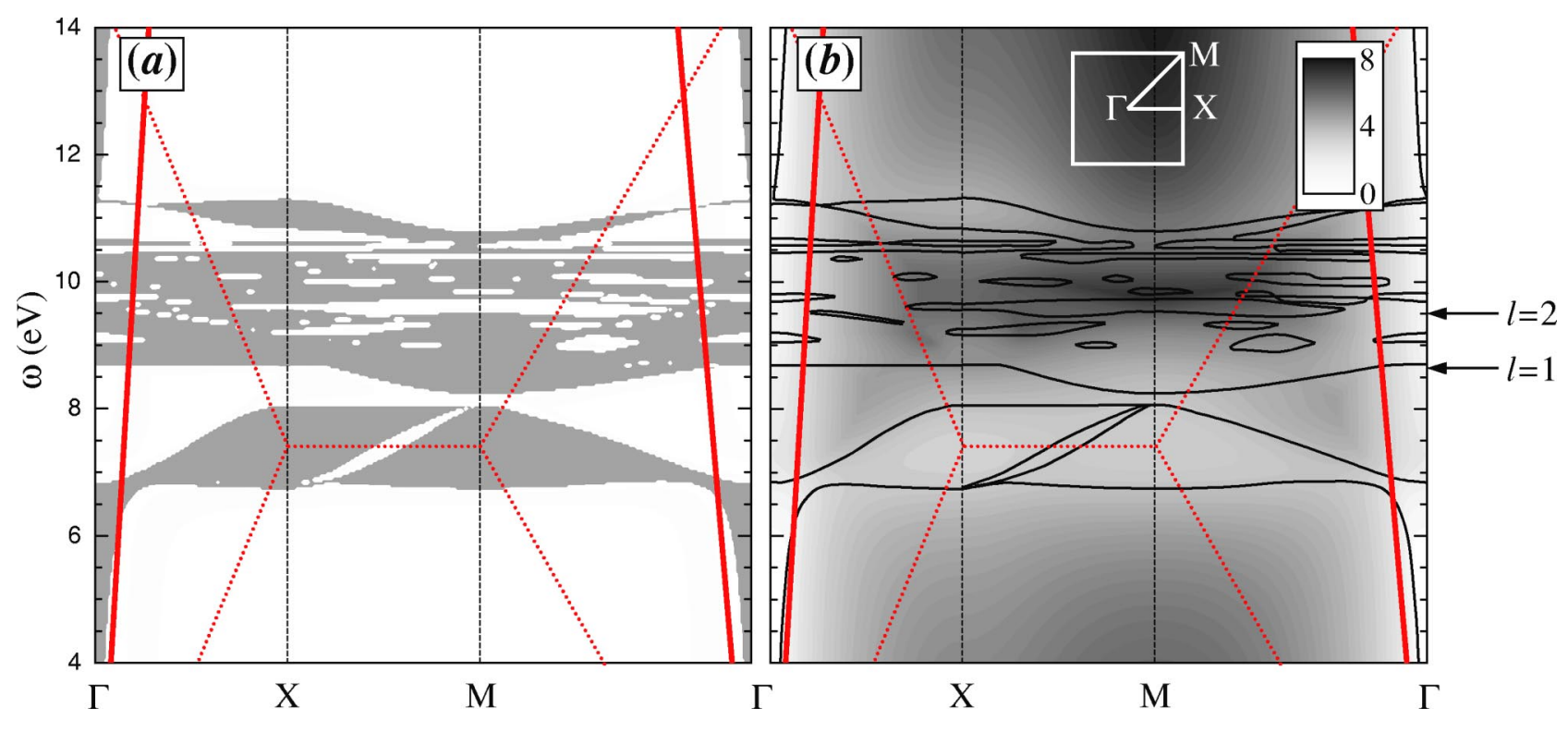

FIG. 2. (color online only) Photonic band structure of one of the crystals considered in Fig. 1, consisting of a simple cubic lattice of aluminum spheres in vacuum with a lattice constant of $5 \mathrm{~nm}$ and a filling fraction of $30 \%$. The figure shows the band structure projected on the (100) surface. The horizontal axis represents the excursion along the points indicated in the inset within the parallel momentum plane. The expression $\epsilon(\omega)=1-\omega_{p}^{2} / \omega(\omega+\mathrm{i} \eta)$ has been used for the aluminum dielectric constant with $\omega_{p}=15 \mathrm{eV}$. When the damping is taken in the $\eta \rightarrow 0^{+}$limit, one obtains regions of allowed electromagnetic propagation, that is, combinations of the energy and the parallel momentum components for which some eigenstates have a real perpendicular momentum. They define the shaded region in (a). However, for a realistic value of the damping parameter $(\eta=1 \mathrm{eV})$, all perpendicular momentum components are complex and they represent evanescent waves within the crystal. The contour plot in (b) represents the lowest value of the imaginary part of the perpendicular momentum multiplied by the lattice constant (i.e., the minimum of $\operatorname{Im}\left\{k_{z} a\right\}$ ) for all eigenstates with a given parallel momentum. The boundaries of the shaded areas of (a) are shown in (b) as continuous curves. Straight lines have been added to both (a) and (b) to represent the speed cones $\omega=\mathbf{Q} \cdot \mathbf{v}$, corresponding to the external field associated with the electron moving at speeds $v=0.4 c$ (thick solid lines) and $v=0.06 c$ (dotted lines).

tained for an electron moving parallel to a homogeneous surface of a material described by such a dielectric function. Several recipes for defining $\epsilon_{\text {eff }}$ for granular materials have been given in the literature, ranging from simple effectivemedium theories like Maxwell-Garnett's ${ }^{28}$ to more elaborate ones that take into account the actual shape of the constituents, both for disordered composites ${ }^{29,30,17}$ and for crystals. ${ }^{29,31-34}$ Among the latter, one finds extensions of the Maxwell-Garnett theory that go beyond dipolar interactions, ${ }^{29,17}$ spectral representations, ${ }^{31,33}$ or direct derivation of the light dispersion relation. ${ }^{32}$ Here, we have used a different method that consists in finding the effective dielectric function $\epsilon_{\text {eff }}$ of the equivalent homogeneous medium that has the same reflectance as the crystal surface: ${ }^{26}$ first, the reflectance coefficients for $s$ - and $p$-polarized light, $r_{s}$ and $r_{p}$, respectively, are calculated for the actual crystal as a function of the angle of incidence $\theta$; then, $\epsilon_{\text {eff }}$ is derived from Fresnel equations, which are conveniently recast for $s$ light as $\epsilon_{\text {eff }}$ $=\sin ^{2} \theta+\cos ^{2} \theta\left[\left(1-r_{s}\right) /\left(1+r_{s}\right)\right]^{2}$, and for $p$ light as a similar but more involved expression; a value of $\epsilon_{\text {eff }}$ averaged over different polarizations and incidence directions is obtained, exhibiting a standard deviation below $1 \%$ in the present case; as a cross check, the reflectance calculated for the actual crystal has been shown to be well reproduced by Fresnel equations with a single average value of $\epsilon_{\text {eff }}(\omega)$ within a $2 \%$ under the present conditions; this procedure is then repeated for each frequency $\omega$ under consideration. The results are represented by the solid curves of Fig. 1(b), where they are compared with Maxwell-Garnett's theory (broken curves). For $f=6.5 \%$ these two models are nearly identical, as Maxwell-Garnett formula gives the dipole of the small isolated sphere correctly. However, for $f=30 \%$ the deviation between both models is significant, since higher momenta are involved in the interaction between neighboring spheres. The magnitude actually represented in the figure is the surface loss function, $\operatorname{Im}\left\{-1 /\left(1+\epsilon_{\text {eff }}\right)\right\}$, which is directly comparable to the loss probability of Fig. 1(a). The agreement in the position of the peaks between the detailed energy-loss calculation and our effective-medium theory is reasonably good, indicating that an effective homogeneous medium describes the solid appropriately within this energy range at $v$ $=0.4 c$; however, the relative weight of the features for $f$ $=30 \%$ changes completely at lower velocities (dotted curve, $v=0.06 c=8.2$ a.u.).

Another example of a crystal that can be represented by an effective dielectric constant is given in Fig. 3, consisting of a simple cubic lattice with the same parameters as in Fig. 1 , except that finite cylinders have been used instead of spheres. As a result, the medium is strongly anisotropic and characterized by different bulk plasmon modes when the electric field is directed parallel or perpendicular to the cylinders [see Figs. 3(c,d)]. The loss probability [Fig. 3(a)] is shown to share most of the features of the surface loss function for anisotropic media [Fig. 3(b)], except for the $7.8 \mathrm{eV}$ peak in the loss spectrum with $f=30 \%$, which might be connected to the proximity effect when the electron starts 

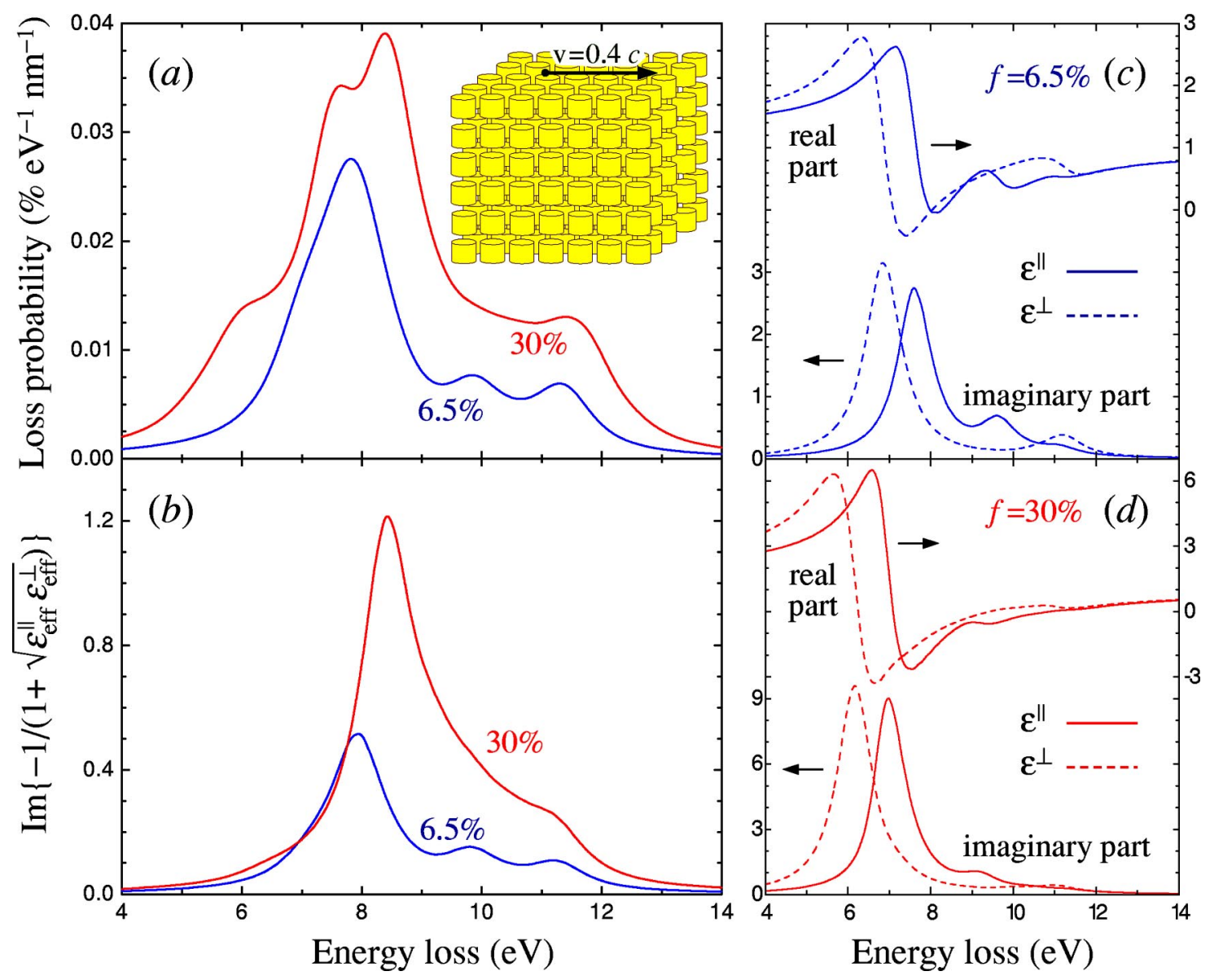

FIG. 3. (color online only) (a) Energy-loss spectra for an electron moving parallel to the [100] direction of the (100) surface of a simple cubic crystal made up of six layers of aluminum cylinders in vacuum with lattice constant $5 \mathrm{~nm}$ and two different filling fractions (see labels). The height of the cylinders is equal to the diameter in all cases. The electron is moving at a distance of $1 \mathrm{~nm}$ from the cylinder surfaces with a velocity $v=0.4 c$. (b) Surface loss function for the same crystal as in (a) using the effective dielectric function obtained from the multiple-scattering method explained in the text. (c) Anisotropic dielectric function for the crystal considered in (a) with a filling fraction of the aluminum of $6.5 \%$. (d) Same as (c), for a filling fraction of $30 \%$.

sensing the nonuniform character of the surface via evanescent waves.

\section{ENERGY LOSS FOR LATTICE CONSTANTS COMPARABLE TO THE WAVELENGTH}

For lattice constants comparable to the wavelength associated to the energy losses under consideration, one can no longer define the effective dielectric constant of an equivalent homogeneous medium. Then, it is useful to relate the loss spectra directly to the photonic band structure. This has been done in Fig. 4 for a crystal of aluminum spheres immersed in a dielectric with $\epsilon=3$. The features of the loss spectra [Fig. 4(c)] seem to be correlated to some extend with the band structure [Figs. 4(a,b)]. In particular, the pseudogaps near $3.2 \mathrm{eV}$ could be the source of a dip in the loss probability. However, this relation is not entirely direct, as can be seen from the presence of a peak at around $2.7 \mathrm{eV}$, whose origin could be found in the coupling of the external electron trajectory to crystal surface modes. More research in this direction is still needed.
Using electrons to analyze the crystal permits exploring regions of the band structure that are not accessible to external light, but that can be reached via the evanescent waves contained in the perturbing field of the electrons. This is the case of Fig. 4 for $v / c=0.5$ [solid curve in Fig. 4(c)], well below the Cherenkov threshold $v / c=1 / \sqrt{3}$, that permits to observe how the peak in the loss spectrum near $2.7 \mathrm{eV}$ results from the coupling with propagating modes of the crystal as a result of the intersection of the external field components [straight solid lines in Fig. 4(a)] and the allowed regions of propagation (shaded areas).

At larger velocities $[v / c=0.75$, dashed curve in Fig. $4(\mathrm{c})$, Cherenkov radiation would be produced in the absence of the crystal, that would result in a loss probability independent of $\omega$ (see little arrow near the horizontal axis of the figure). This is clearly seen in the big overlap of the external field with the regions of propagation within the crystal inside the low-energy region results in an enhanced loss probability, as compared to $v / c=0.5$. However, the presence of the crystal modulates the loss spectrum.

Part of the energy lost by the electron must be converted 

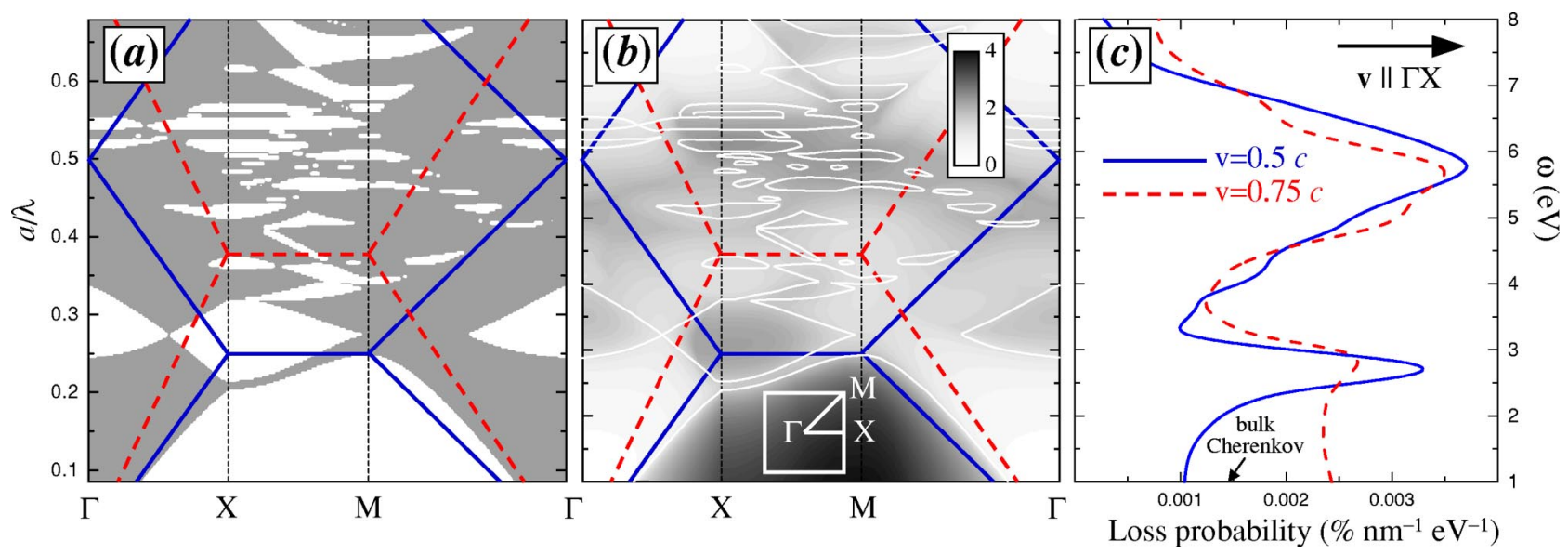

FIG. 4. (a),(b) Photonic band structure projected on the (100) surface and represented as in Figs. 2(a,b) for an fcc lattice of aluminum spheres surrounded by a medium of dielectric function equal to 3. The filling fraction of the spheres is $20 \%$ and the lattice constant is $a$ $=105.2 \mathrm{~nm}$, comparable to the wavelength $\lambda$ (see scale on the left-hand side of the figure). Straight lines have been added to both (a) and (b) to represent the speed cones $\omega=\mathbf{Q} \cdot \mathbf{v}$, corresponding to the external field associated with the electron moving at speeds $v=0.5 c$ (solid lines) and $v=0.75 c$ (thick dashed lines). (c) Electron energy-loss spectra for an electron moving with velocity $v=0.5 c$ (solid curve) and $v=0.75 \mathrm{c}$ (broken curve) at a distance of $3.2 \mathrm{~nm}$ from the surface of the outermost spheres of a semi-infinite crystal like that considered in (b). The trajectory is directed along the $[100]$ direction $(\Gamma X)$.

into the so-called Smith-Purcell radiation. ${ }^{21,10}$ In particular, when transparent materials are used to build the crystals, the light-emission probability must coincide with the energy-loss probability. For a crystal of finite thickness, like the one considered in Fig. 5, consisting of 8 (111) layers of an inverted Si opal $(\epsilon=11.9)$ with a filling fraction of $67 \%$, part of this emission occurs towards the side of the crystal opposite to the electron trajectory (here, the electron is taken to be moving parallel to the crystal surface). The intensity of the emitted light [Fig. 5(c)] has been calculated from the integral of the Poynting vector far from the crystal (see Ref. 23 for more details), and it presents a strong dip near $a / \lambda \approx 0.75$, where $a=1220 \mathrm{~nm}$ is the lattice constant. The region of emission depletion is actually contained within a full band gap of the infinite crystal [see Fig. 5(a)]; this is also seen in the transmission of light incident on the crystal both normal to the surface [dashed curve in Fig. 5(b)] or with an angle corresponding to the Cherenkov radiation produced by the electron in the plane defined by the trajectory and the surface normal (solid curve).

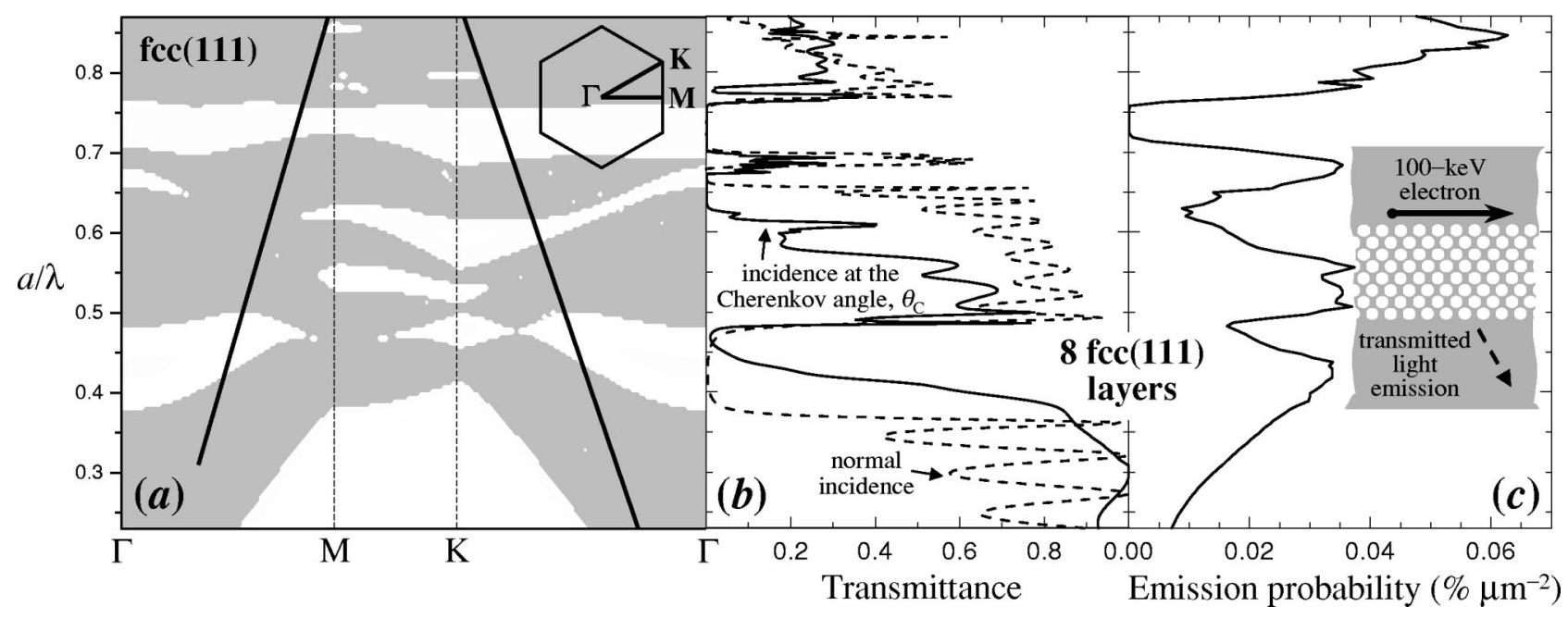

FIG. 5. (a) Photonic band structure projected on the (111) surface for an fcc lattice of air spherical voids in Si $(\epsilon=11.9)$. The radius of the spheres is 0.342 times the lattice constant $a$. (b) Dashed curve: transmittance of light incident normal to eight layers of the crystal considered in (a). Solid curve: transmittance for an angle of incidence corresponding to the Cherenkov angle $\theta_{c}=68.1^{\circ}$ for a $100-\mathrm{keV}$ electron moving parallel to the same crystal. The crystal is surrounded by Si on both surfaces. (c) Probability of emitting light on the opposite side of the crystal with respect to the electron trajectory. The distance from the latter to the sphere surfaces is $876 \mathrm{~nm}$ and the lattice constant is $a=1220 \mathrm{~nm}$. The probability is given per unit of path length in microns and per unit of emitted-photon wavelength also in microns. The region of frequency and momentum where the field of the electron inside a homogeneous infinite Si medium takes nonzero values is represented by thick solid lines in (a). 


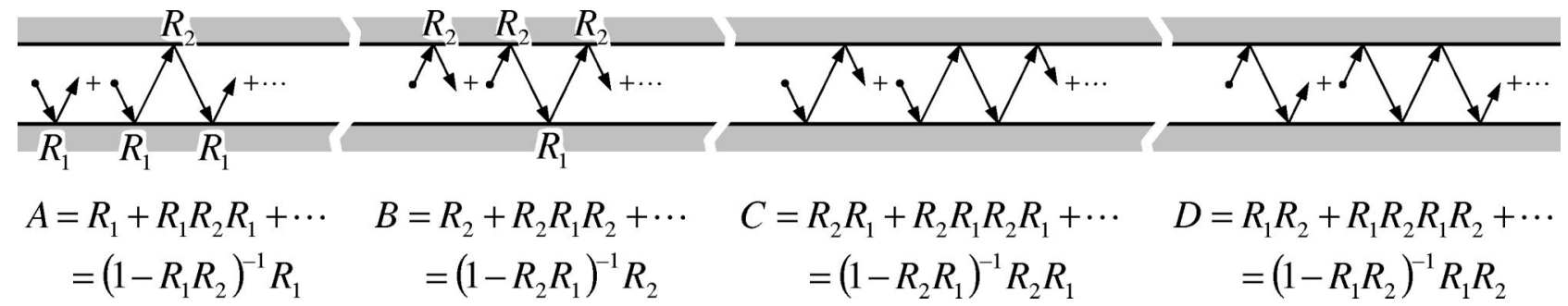

FIG. 6. Schematic representation of the construction of the electric field in the bulk of a photonic crystal in terms of the reflectance of two semi-infinite crystals and definition of the matrices $A, B, C$, and $D$ used in Eq. (7). See Sec. V for more details.

\section{ENERGY LOSS IN THE BULK OF A CRYSTAL}

So far we have considered an electron moving in front of the surface of a photonic crystal. When the electron is moving in the bulk of an infinite crystal, the electric field can also be written in terms of reflectance coefficients if the trajectory is contained in a low-index plane that does not intersect any of the crystal objects. The reflectance in question is that of the lower and upper semi-infinite crystals into which the entire crystal is divided by the noted plane. The corresponding reflectance matrices will be denoted $R_{1}$ and $R_{2}$, respectively. They contain the amplitudes of reflected beams, labeled by vectors $\mathbf{G}$ of the 2D reciprocal lattice of the plane under consideration. In particular, for a fixed value of $\mathbf{Q}, R_{1, \mathbf{G G}}^{\sigma \sigma \prime}$, is


$R_{2, \mathbf{G G}}^{\sigma \sigma \prime}$, connects $\mathbf{K}_{\mathbf{Q}+\mathbf{G}}^{+}$wave components with waves reflected from the upper surface with momentum $\mathbf{K}_{\mathbf{Q}+\mathbf{G}}^{-}$.

This is represented schematically in Fig. 6, where the reflectance matrices of the lower and upper semi-infinite crystals are $R_{1}$ and $R_{2}$, respectively, and upwards (downwards) arrows represent waves of momentum $\mathbf{K}_{\mathbf{Q}+\mathbf{G}}^{+}\left(\mathbf{K}_{\mathbf{Q}+\mathbf{G}}^{-}\right)$. The upper and lower surfaces have been separated in the figure for the sake of clarity, but they will be considered to lie on the same plane (a plane that contains the electron trajectory) in what follows.
Using Eq. (5) for the loss probability and a straightforward extension of Eq. (4) for the reflected electric field including all terms of Fig. 6, the loss probability per unit of path length averaged over all impact parameters parallel to the crystal surfaces is found to be

$$
\begin{aligned}
P_{r}(\omega)= & \frac{1}{\pi v^{2}} \int \frac{d Q_{y}}{Q^{2}} \operatorname{Im}\left\{\left[A_{00}^{p p}+B_{00}^{p p}-C_{00}^{p p}-D_{00}^{p p}\right.\right. \\
& +\epsilon_{h} \mu_{h}\left(\frac{Q_{y} v}{\Gamma_{Q} c}\right)^{2}\left(A_{00}^{s s}+B_{00}^{s s}+C_{00}^{s s}+D_{00}^{s s}\right) \\
& +\frac{\mathrm{i} Q_{y} k_{h} v}{\omega \Gamma_{Q}}\left(A_{00}^{s p}-B_{00}^{s p}+C_{00}^{s p}-D_{00}^{s p}-A_{00}^{p s}\right. \\
& \left.\left.\left.+B_{00}^{p s}+C_{00}^{p s}-D_{00}^{p s}\right)\right] \frac{\Gamma_{Q}}{\epsilon_{h}}\right\},
\end{aligned}
$$

where the matrices $A_{\mathbf{G G}^{\prime}}^{\sigma \sigma^{\prime}}, B_{\mathbf{G G}^{\prime}}^{\sigma \sigma^{\prime}}, C_{\mathbf{G G}^{\prime}}^{\sigma \sigma^{\prime}}$, and $D_{\mathbf{G G}^{\prime}}^{\sigma \sigma^{\prime}}$ are defined in Fig. 6, and only the elements $\mathbf{G}=\mathbf{G}^{\prime}=0$ enter this expression.

The above formula has been applied to calculate the energy-loss probability for an electron moving in between

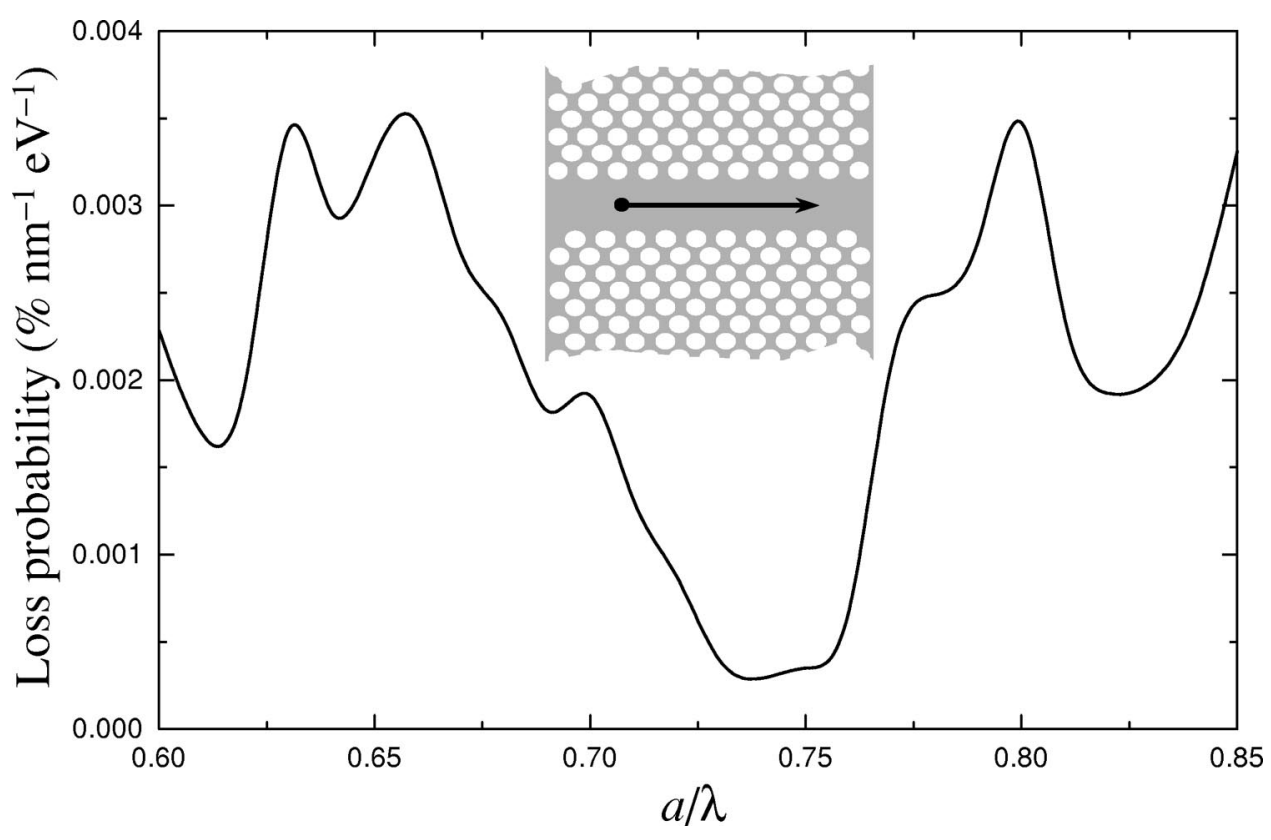

FIG. 7. Energy-loss probability for a $100-\mathrm{keV}$ electron traveling parallel to two photonic crystals with the same parameters as in Fig. 5(c). The spacing between the two crystals is $300 \mathrm{~nm}$, which is filled with $\mathrm{Si}$ (this is exaggerated in the inset for the sake of clarity). The electron is traveling at the same distance from the two crystals. 
two parallel crystal slabs of 32 layers each surrounded by $\mathrm{Si}$, as shown in Fig. 7. The crystal parameters are the same as in Fig. 5(c), so that an absolute band gap shows up near $a / \lambda$ $=0.75$. The electron energy $(100 \mathrm{keV})$ is large enough to produce Cherenkov light, which must be confined in between the two crystals for wavelengths lying in the gap region. This is actually observed as a pronounced dip, connected to the fact that no electromagnetic modes can be created that escape through the crystals, except for a small transmission due to their finite thickness. However, some radiation can escape through modes that are trapped in the slab formed by the two crystals, so that the loss probability does not actually reach a zero value in the dip.

\section{CONCLUDING REMARKS}

The energy loss of fast electrons interacting with photonic crystals has been calculated for dielectric, metallic, and metalodielectric systems.

When the wavelength of the radiation associated to the energy loss is much larger than the lattice constant, the crystal can be regarded as a continuous medium, characterized by an effective dielectric function, as it has been shown for crystals of small aluminum spheres in Fig. 1 and small finite cylinders in Fig. 3. The effective medium is highly anisotropic in the latter case. The effective dielectric function has been calculated in both cases from the reflectance coefficient of the crystal, and this has been shown to contain most of the information needed to understand the calculated energy-loss spectra.

When the lattice constant is comparable to the wavelength, the concept of an equivalent effective continuous me- dium is no longer valid, and one has to rely on the detailed band structure of the crystal to understand the loss spectra [Figs. 4, 5, and 7].

Finally, the interaction of the electron with the crystal produces Smith-Purcell radiation, which contributes to the energy loss. If the crystal is composed of nonabsorbing materials, the energy-loss probability and the light-emission probability must coincide, as shown in Fig. 5 for an inverted Si opal, in which case the light emitted after transmission through the crystal shows dips that are directly connected to the presence of photonic band gaps. For electrons moving in a region surrounded by photonic crystals a dip is also observed in the loss probability within the gap energies (Fig. 7).

Our hope is that the present work can provide a stimulus to use fast electrons in the analysis of photonic crystals as a way to bring a source of evanescent radiation (the electromagnetic field of the electron in vacuum) into close contact with the crystal and also to probe regions of the crystal that would not be easily accessible to other sources of external electromagnetic radiation.

\section{ACKNOWLEDGMENTS}

The authors would like to thank P. M. Echenique for helpful and stimulating discussions. This work has been supported in part by the Basque Departamento de Educación, Universidades e Investigación, the University of the Basque Country UPV/EHU (Contract No. 00206.215-13639/2001), and the Spanish Ministerio de Ciencia y Tecnología (Contract No. MAT2001-0946).
*Email address: jga@sw.ehu.es

${ }^{1}$ L.D. Marks, Solid State Commun. 43, 727 (1982).

${ }^{2}$ J.M. Cowley, Phys. Rev. B 25, 1401 (1982).

${ }^{3}$ A. Howie and R.H. Milne, Ultramicroscopy 18, 427 (1985).

${ }^{4}$ R.H. Ritchie and A. Howie, Philos. Mag. A 58, 753 (1988).

${ }^{5}$ P.M. Echenique and J.B. Pendry, J. Phys. C 8, 2936 (1975).

${ }^{6}$ T.L. Ferrell and P.M. Echenique, Phys. Rev. Lett. 55, 1526 (1985)

${ }^{7}$ A. Rivacoba, N. Zabala, and P.M. Echenique, Phys. Rev. Lett. 69, 3362 (1992).

${ }^{8}$ B.T. Draine and P.J. Flatau, J. Opt. Soc. Am. A 11, 1491 (1994).

${ }^{9}$ F.J. García de Abajo and A. Howie, Phys. Rev. Lett. 80, 5180 (1998); Phys. Rev. B 65, 115418 (2002).

${ }^{10}$ F.J. García de Abajo, Phys. Rev. Lett. 82, 2776 (1999); Phys. Rev. B 59, 3095 (1999); Phys. Rev. E 61, 5743 (2000).

${ }^{11}$ E. Yablonovitch, Phys. Rev. Lett. 58, 2059 (1987).

${ }^{12}$ S. John and J. Wang, Phys. Rev. Lett. 64, 2418 (1990).

${ }^{13}$ S.D. Hart, G.R. Maskaly, B. Temelkuran, P.H. Prideaux, J.D. Joannopoulos, and Y. Fink, Science 296, 510 (2002).

${ }^{14}$ S.-Y. Lin, E. Chow, V. Hietala, P.R. Villeneuve, and J.D. Joannopoulos, Science 282, 274 (1998).

${ }^{15}$ L. Fu, P.B. Macedo, and L. Resca, Phys. Rev. B 47, 13818 (1993).
${ }^{16}$ L. Fu and L. Resca, Phys. Rev. B 47, 16194 (1993).

${ }^{17}$ R.G. Barrera and R. Fuchs, Phys. Rev. B 52, 3256 (1995).

${ }^{18}$ C.I. Mendoza, R.G. Barrera, and R. Fuchs, Phys. Rev. B 57, 11193 (1998).

${ }^{19}$ J.B. Pendry and L. Martín-Moreno, Phys. Rev. B 50, 5062 (1994).

${ }^{20}$ J.B. Pendry and A. MacKinnon, Phys. Rev. Lett. 69, 2772 (1992).

${ }^{21}$ S.J. Smith and E.M. Purcell, Phys. Rev. 92, 1069 (1953).

${ }^{22}$ K. Ohtaka and S. Yamaguti, Opt. Spectrosc. 91, 506 (2001).

${ }^{23}$ L. A. Blanco and F. J. García de Abajo, Surf. Sci. (to be published).

${ }^{24}$ Within the retarded response formalism used here, the imaginary part of $k_{h}$ is positive. Thus, $\operatorname{Re}\left\{\Gamma_{Q}\right\}$ and $\operatorname{Im}\left\{\Gamma_{Q}\right\}$ must have opposite signs. Now, with our choice of $\operatorname{Re}\left\{\Gamma_{Q}\right\}>0$, and for $\epsilon_{h}$ positive and real, in which case the imaginary part of $k_{h}$ is infinitesimal (e.g., in vacuum), one can distinguish too kinds of waves: (i) for $Q>k_{h}, \Gamma_{Q}$ is real and positive and then $\mathbf{K}_{\mathbf{Q}}^{+}$ represents the momentum of an evanescent plane wave that decays with increasing $z$; (ii) for $Q<k_{h}, \Gamma_{Q}$ is imaginary and $\mathbf{K}_{\mathbf{Q}}^{+}$ is the momentum of a propagating plane wave that moves without attenuation towards the $z>0$ direction.

${ }^{25}$ N. Stefanou, V. Yannopapas, and A. Modinos, Comput. Phys. Commun. 113, 49 (1998); 132, 189 (2000). 
${ }^{26}$ F. J. García de Abajo (unpublished).

${ }^{27}$ J. D. Jackson, Classical Electrodynamics (Wiley, New York, 1975).

${ }^{28}$ J.C. Maxwell-Garnett, Philos. Trans. R. Soc. London, Ser. A 203 , 385 (1904); 205, 237 (1906)

${ }^{29}$ W. Lamb, D.M. Wood, and N.W. Ashcroft, Phys. Rev. B 21, 2248 (1980).

${ }^{30}$ R.I. Cukier, J. Karkheck, S. Kumar, and S.Y. Sheu, Phys. Rev. B
41, 1630 (1990).

${ }^{31}$ D.J. Bergman and K.-J. Dunn, Phys. Rev. B 45, 13262 (1992).

${ }^{32}$ L. Martín-Moreno and J.B. Pendry, Nucl. Instrum. Methods Phys. Res. B 96, 565 (1995).

${ }^{33}$ H. Ma, B. Zhang, W.Y. Tam, and P. Sheng, Phys. Rev. B 61, 962 (2000).

${ }^{34}$ D.R. Smith, S. Schultz, P. Markos, and C.M. Soukoulis, Phys. Rev. B 65, 195104 (2002). 